\title{
Densities, Viscosities and Self-Diffusion Coefficients of Several Polyethylene Glycols
}

\author{
Markus M. Hoffmann, ${ }^{* 1}$ Joseph D. Kealy, ${ }^{1}$ Torsten Gutmann, ${ }^{2}$ and Gerd Buntkowsky*2 \\ ${ }^{1}$ Department of Chemistry and Biochemistry, State University of New York College at Brockport, \\ Brockport, NY, 14420, USA \\ ${ }^{2}$ Institute of Physical Chemistry, Technical University Darmstadt, Alarich-Weiss-Straße 8, D-64287 \\ Darmstadt, Germany \\ Corresponding Authors* \\ Markus M. Hoffmann \\ E-mail: mhoffman@brockport.edu
}

Gerd Buntkowsky

E-mail: gerd.buntkowsky@chemie.tu-darmstadt.de 
Table S1: Standard Deviations for Reported Viscosity Measurements in $\mathrm{mPa} \cdot \mathrm{s}^{\mathrm{a}}$

\begin{tabular}{lcccccc}
\hline$T / \mathrm{K}$ & \multicolumn{7}{c}{} \\
\hline \multicolumn{7}{c}{ Acros PEG200 } \\
$w / 10^{-6}$ & 1834 & 6963 & 13136 & 16528 & 33512 & 87088 \\
298.15 & 0.431 & 0.857 & 0.248 & 0.138 & 0.112 & 0.068 \\
308.15 & 0.101 & 0.081 & 0.085 & 0.099 & 0.057 & 0.020 \\
318.15 & 0.078 & 0.381 & 0.062 & 0.075 & 0.019 & 0.007 \\
328.15 & 0.055 & & 0.057 & 0.055 & 0.001 & 0.002 \\
338.15 & 0.073 & 0.006 & 0.078 & 0.080 & 0.004 & 0.008 \\
348.15 & & 0.003 & & & 0.008 & \\
358.15 & & 0.002 & & & &
\end{tabular}

Rigaku PEG200

$\begin{array}{lrrrr}w / 10^{-6} & 8781 & 12318 & 13837 & 18248 \\ 298.15 & 0.452 & 0.087 & 0.380 & 0.347 \\ 308.15 & 0.093 & 0.105 & 0.120 & 0.053 \\ 318.15 & 0.178 & 0.083 & 0.091 & 0.508 \\ 328.15 & 0.006 & 0.066 & 0.074 & 0.006 \\ 338.15 & 0.006 & 0.093 & 0.098 & 0.006 \\ 348.15 & 0.032 & & & 0.003 \\ 358.15 & 0.004 & & & 0.009\end{array}$

Acros PEG400

$\begin{array}{lrrrrr}w / 10^{-6} & 3315 & 8408 & 13089 & 16461 & 17028 \\ 298.15 & 0.205 & 0.368 & 0.212 & 0.313 & 0.132 \\ 308.15 & 0.046 & 0.180 & 0.081 & 0.017 & 0.091 \\ 318.15 & 0.035 & 0.076 & 0.010 & 0.021 & 0.035 \\ 328.15 & 0.010 & 0.006 & 0.050 & 0.012 & 0.010 \\ 338.15 & 0.015 & 0.006 & 0.015 & 0.012 & 0.006 \\ 348.15 & 0.006 & 0.010 & 0.023 & 0.006 & 0.010 \\ 358.15 & 0.011 & & 0.003 & 0.013 & 0.004\end{array}$

$\begin{array}{lllll}w / 10^{-6} & 1030 & 3921 & 7607 & 12833\end{array}$

$\begin{array}{lllll}298.15 & 0.386 & 0.102 & 0.122 & 0.104\end{array}$

$\begin{array}{lllll}308.15 & 0.272 & 0.049 & 0.010 & 0.055\end{array}$

$\begin{array}{lllll}318.15 & 0.211 & 0.025 & 0.010 & 0.050\end{array}$

$\begin{array}{lllll}328.15 & 0.010 & 0.012 & 0.010 & 0.012\end{array}$

$\begin{array}{lllll}338.15 & 0.010 & 0.006 & 0.006 & 0.006\end{array}$

$\begin{array}{lllll}348.15 & 0.002 & 0.002 & 0.002 & 0.002\end{array}$

$\begin{array}{lllll}358.15 & 0.002 & 0.002 & 0.003 & 0.003\end{array}$

$x_{1}=0.424, x_{2}=0.576$

$\begin{array}{lrrrr}w / 10^{-6} & 1235 & 3632 & 8847 & 12791 \\ 298.15 & 0.075 & 0.154 & 0.134 & 0.098\end{array}$




\begin{tabular}{|c|c|c|c|c|}
\hline 308.15 & 0.096 & 0.012 & 0.065 & 0.021 \\
\hline 318.15 & 0.083 & 0.012 & 0.062 & 0.035 \\
\hline 328.15 & 0.051 & 0.006 & 0.020 & 0.006 \\
\hline 338.15 & 0.074 & 0.006 & & \\
\hline 348.15 & & 0.003 & 0.006 & 0.003 \\
\hline 358.15 & & 0.002 & & \\
\hline \multicolumn{5}{|c|}{$x_{1}=0.620, x_{2}=0.380$} \\
\hline$w / 10^{-6}$ & 1462 & 3638 & 8081 & 14417 \\
\hline 298.15 & 0.328 & 0.120 & 0.042 & 0.079 \\
\hline 308.15 & 0.057 & 0.040 & 0.036 & 0.006 \\
\hline 318.15 & 0.069 & 0.012 & 0.027 & 0.025 \\
\hline 328.15 & 0.012 & 0.006 & 0.006 & 0.006 \\
\hline 338.15 & 0.006 & 0.000 & 0.006 & 0.006 \\
\hline 348.15 & 0.006 & 0.004 & 0.016 & 0.002 \\
\hline 358.15 & 0.007 & 0.001 & & \\
\hline \multicolumn{5}{|c|}{$x_{1}=0.661, x_{2}=0.339$} \\
\hline$w / 10^{-6}$ & 1517 & 4631 & 8906 & \\
\hline 298.15 & 0.059 & 0.096 & 0.063 & \\
\hline 308.15 & 0.106 & 0.006 & 0.051 & \\
\hline 318.15 & 0.085 & & 0.012 & \\
\hline 328.15 & 0.066 & 0.010 & 0.020 & \\
\hline 338.15 & 0.092 & 0.006 & 0.006 & \\
\hline 348.15 & & 0.003 & 0.006 & \\
\hline 358.15 & & 0.002 & & \\
\hline \multicolumn{5}{|c|}{$x_{1}=0.752, x_{2}=0.248$} \\
\hline$w / 10^{-6}$ & 1651 & 5096 & 10593 & 13132 \\
\hline 298.15 & 0.239 & 0.115 & 0.113 & 0.054 \\
\hline 308.15 & 0.123 & 0.010 & 0.046 & 0.040 \\
\hline 318.15 & 0.086 & 0.010 & 0.031 & 0.015 \\
\hline 328.15 & 0.083 & 0.006 & 0.006 & 0.010 \\
\hline 338.15 & 0.122 & 0.004 & 0.003 & 0.003 \\
\hline 348.15 & & 0.030 & 0.002 & 0.002 \\
\hline 358.15 & & 0.002 & & \\
\hline
\end{tabular}

${ }^{a}$ Empty entries are due to either absence of measurements or because instrument settings were set to only show measurement average. Relative standard uncertainty of mass fraction $w$ is 0.10 . Standard uncertainty of temperature is $0.02 \mathrm{~K}$, and for the mole fraction, $x$, of tri- (1) and hexaetheylene glycol (2) is 0.001 . 
Table S2: Comparison of Viscosity Measurements in $\mathrm{mPa} \cdot \mathrm{s}$ at $(0.10 \pm 0.01)$

$\mathrm{MPa}$ From Two Instruments on Same Triethylene Glycol Sample as well as

with prior measurements.

\begin{tabular}{|c|c|c|c|c|c|c|}
\hline $\mathrm{T} / \mathrm{K}^{\mathrm{a}}$ & AMVn & Lovis & $\%$ difference & average & $\begin{array}{c}\text { Hoffmann et } \\
\text { al. }^{\mathrm{b}}\end{array}$ & $\% \mathrm{RD}^{\mathrm{c}}$ \\
\hline 298.15 & 38.69 & 38.28 & 1.06 & 38.49 & 37.1 & 3.73 \\
\hline 308.15 & 24.15 & 24.33 & -0.73 & 24.24 & 23.4 & 3.59 \\
\hline 318.15 & 16.06 & 16.18 & -0.79 & 16.12 & 15.8 & 2.03 \\
\hline 328.15 & 11.21 & 11.24 & -0.21 & 11.23 & 11.2 & 0.22 \\
\hline 338.15 & 8.13 & 8.16 & -0.45 & 8.15 & 8.2 & -0.67 \\
\hline 348.15 & 6.25 & 6.18 & 1.15 & 6.22 & 6.4 & -2.89 \\
\hline
\end{tabular}

a Standard uncertainty of temperature is $0.02 \mathrm{~K}$

${ }^{\mathrm{b}}$ Results from the author's laboratory recently reported in Hoffmann et al. ${ }^{1}$

${ }^{\mathrm{c}}$ Based on these \%RD values, the relative standard uncertainty of the viscosities is estimated to be 0.03 which amounts to about $1 \mathrm{mPa}$ s for low temperatures where measured viscosities are highest and about $0.2 \mathrm{mPa} \cdot \mathrm{s}$ at the highest temperatures where measured viscosities are lowest.

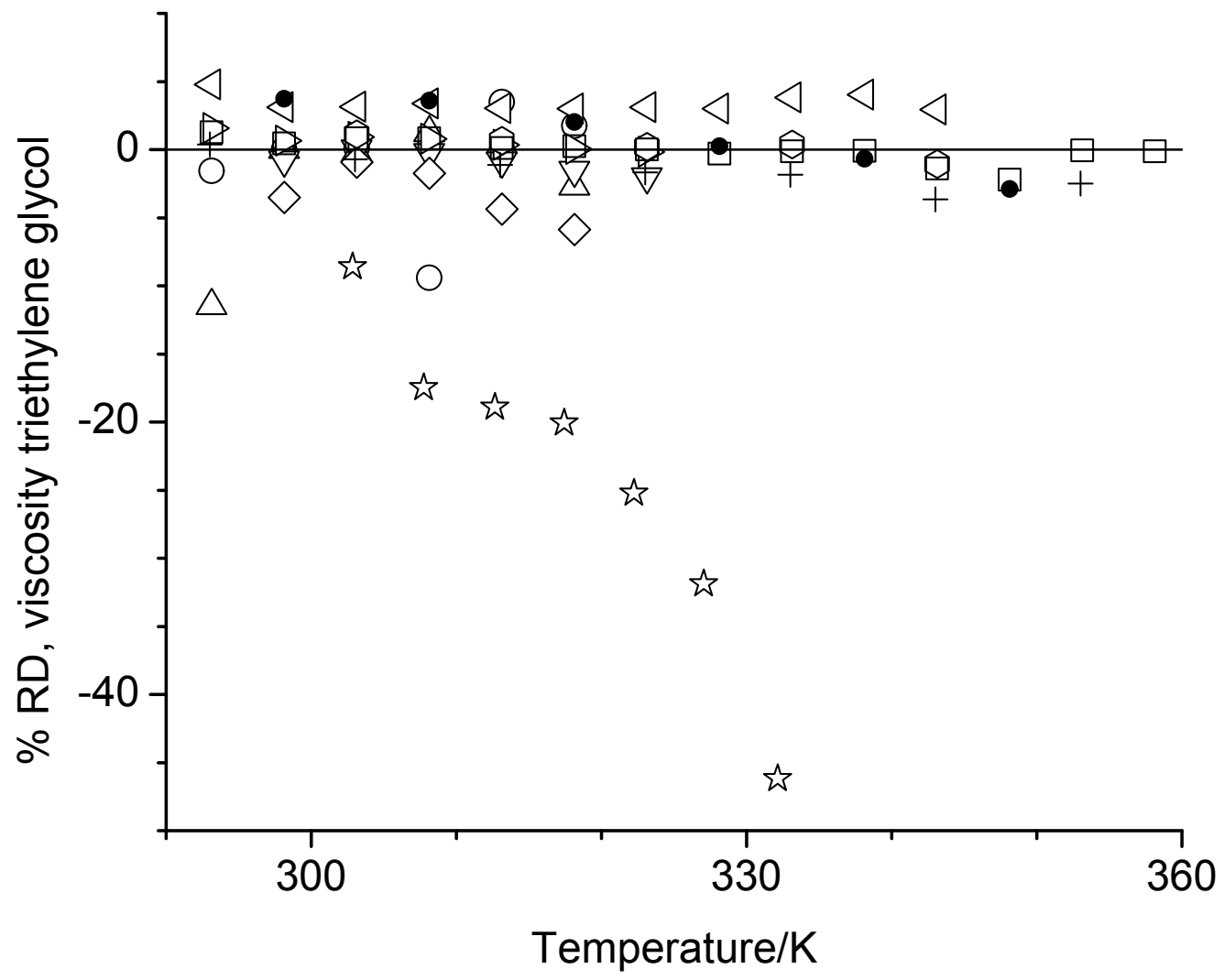

Figure S1. \% relative deviation (\%RD) of triethylene glycol viscosity results from Carvalho et al. ${ }^{2}(\square)$, Hao et al. ${ }^{3}(\bigcirc)$, Qiao et al. ${ }^{4}(\triangle)$, Begum et al. ${ }^{5}(\nabla)$, Guo et al. ${ }^{6}(\diamond)$, Ghaedi et al. ${ }^{7}(\square)$, Almasi ${ }^{8}(\diamond)$,

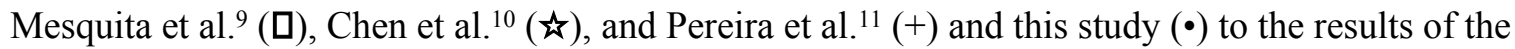
authors's laboratory previously reported in Hoffmann et al. ${ }^{1}$ 
Table S3: Gas Chromatography Mole Fraction Analysis of Ethylene Glycol Oligomers, H-[O-CH$\left.-\mathrm{CH}_{2}\right]_{\mathrm{n}}-$ OH, in PEG 200 from Different Vendors

\begin{tabular}{lrrrrrrrrrr}
\hline & & Alpha & Dow & Dow & \multicolumn{1}{c}{ TLC } \\
$\mathrm{N}$ & Acros & Aesar & batch 1 & batch 2 & Rigaku & Spectrum & America & Average & Stdev & Calc. $^{\text {a }}$ \\
\hline 2 & 0.051 & 0.035 & 0.030 & 0.032 & 0.031 & 0.032 & 0.031 & 0.035 & 0.007 & 0.066 \\
3 & 0.243 & 0.228 & 0.213 & 0.218 & 0.216 & 0.220 & 0.220 & 0.223 & 0.010 & 0.199 \\
4 & 0.312 & 0.327 & 0.321 & 0.319 & 0.318 & 0.318 & 0.324 & 0.320 & 0.005 & 0.315 \\
5 & 0.225 & 0.244 & 0.251 & 0.252 & 0.251 & 0.250 & 0.251 & 0.246 & 0.010 & 0.262 \\
6 & 0.121 & 0.123 & 0.134 & 0.131 & 0.134 & 0.131 & 0.128 & 0.129 & 0.005 & 0.115 \\
7 & 0.047 & 0.043 & 0.050 & 0.048 & 0.049 & 0.048 & 0.045 & 0.047 & 0.003 & 0.026 \\
\hline AMW $^{\mathrm{b}}$ & 205.8 & 208.4 & 211.6 & 210.7 & 211.4 & 210.7 & 210.1 & 209.8 & & 201.6 \\
\hline
\end{tabular}

${ }^{a}$ Calculated from a Gaussian distribution function $p(\mathrm{n})=(\sigma \sqrt{2 \pi})^{-1} e^{-(\mathrm{n}-\overline{\mathrm{n}})^{2} /\left(2 \sigma^{2}\right)}$, with $\mathrm{n}=4.21272$ and sigma $=1.24882$ resulting in a standard deviation of the fit to the data of 0.025 for the mole fraction, which we take as an estimate for the standard uncertainty of the mole fractions in this table.

${ }^{\mathrm{b}}$ Average molar weight in $\mathrm{g} \cdot \mathrm{mol}^{-1}$. 
Table S4: Arrhenius Fits to the PEG ${ }^{a}$ Self-Diffusion Data Based on the $\mathrm{CH}_{2} \mathrm{OH}$ Signal

${ }^{a}$ Includes binary mixtures of triethyleneglycol (1) and hexaethyleneglycol (2)

$\mathrm{b}$ Standard deviation of linear least square fit of logarithmic form of the Arrhenius law to the experimental data. 
Table S5: Interpolated Self-Diffusion Coefficients in $10^{-10} \mathrm{~m}^{2} \cdot \mathrm{s}^{-1} \mathrm{PEGs}^{\mathrm{a}}$

\begin{tabular}{|c|c|c|c|c|c|c|}
\hline$T / \mathrm{K}$ & & & & & slope & intercept \\
\hline \multicolumn{7}{|c|}{ PEG200 Acros } \\
\hline$w / 10^{-6}$ & 1834 & 6963 & 13136 & 16528 & & \\
\hline 298.15 & 0.289 & 0.276 & 0.270 & 0.274 & $-1.2 \pm 0.8$ & $0.28 \pm 0.01$ \\
\hline 308.15 & 0.423 & 0.405 & 0.398 & 0.403 & $-1.5 \pm 1.1$ & $0.42 \pm 0.01$ \\
\hline 318.15 & 0.594 & 0.609 & 0.601 & 0.614 & $1.3 \pm 0.8$ & $0.60 \pm 0.01$ \\
\hline 328.15 & 0.811 & 0.858 & 0.848 & 0.872 & $4.2 \pm 2.0$ & $0.82 \pm 0.02$ \\
\hline 338.15 & 1.118 & 1.219 & 1.207 & 1.251 & $9.4 \pm 4.3$ & $1.14 \pm 0.03$ \\
\hline 348.15 & 1.468 & 1.645 & 1.631 & 1.700 & $16.5 \pm 7.5$ & $1.51 \pm 0.06$ \\
\hline 358.15 & 1.883 & 2.161 & 2.146 & 2.250 & $26.2 \pm 11.8$ & $1.94 \pm 0.09$ \\
\hline \multicolumn{7}{|c|}{ PEG200 Rigaku } \\
\hline$w / 10^{-6}$ & 8781 & 12318 & 13837 & 18248 & & \\
\hline 298.15 & 0.274 & 0.276 & 0.272 & 0.271 & $-0.3 \pm 0.2$ & $0.28 \pm 0.01$ \\
\hline 308.15 & 0.419 & 0.416 & 0.417 & 0.411 & $-0.6 \pm 0.2$ & $0.42 \pm 0.01$ \\
\hline 318.15 & 0.616 & 0.602 & 0.611 & 0.612 & $0.1 \pm 0.8$ & $0.61 \pm 0.01$ \\
\hline 328.15 & 0.862 & 0.833 & 0.867 & 0.867 & $1.5 \pm 2.0$ & $0.85 \pm 0.02$ \\
\hline 338.15 & 1.251 & 1.193 & 1.242 & 1.277 & $3.8 \pm 4.1$ & $1.22 \pm 0.03$ \\
\hline 348.15 & 1.798 & 1.691 & 1.686 & 1.859 & $6.6 \pm 10.7$ & $1.72 \pm 0.08$ \\
\hline 358.15 & 1.926 & 1.808 & 2.229 & 1.996 & $14.5 \pm 22.4$ & $1.90 \pm 0.17$ \\
\hline \multicolumn{7}{|c|}{ PEG400 } \\
\hline$w / 10^{-6}$ & 3747 & 8408 & 13089 & 16461 & & \\
\hline 298.15 & 0.143 & 0.131 & 0.124 & 0.114 & $-2.3 \pm 0.3$ & $0.14 \pm 0.01$ \\
\hline 308.15 & 0.200 & 0.200 & 0.185 & 0.181 & $-1.8 \pm 0.5$ & $0.20 \pm 0.01$ \\
\hline 318.15 & 0.285 & 0.295 & 0.281 & 0.297 & $0.6 \pm 0.9$ & $0.29 \pm 0.01$ \\
\hline 328.15 & 0.386 & 0.419 & 0.406 & 0.456 & $5.2 \pm 1.9$ & $0.38 \pm 0.01$ \\
\hline 338.15 & 0.504 & 0.568 & 0.553 & 0.657 & $11.5 \pm 3.4$ & $0.50 \pm 0.03$ \\
\hline 348.15 & 0.674 & 0.798 & 0.782 & 0.986 & $23.9 \pm 6.2$ & $0.66 \pm 0.05$ \\
\hline 358.15 & 0.868 & 1.079 & 1.055 & 1.402 & $40.9 \pm 10.5$ & $0.84 \pm 0.08$ \\
\hline \multicolumn{7}{|c|}{$x_{1}=0.197, x_{2}=0.803$} \\
\hline$w / 10^{-6}$ & 1030 & 3921 & 7606 & 12833 & & \\
\hline 298.15 & 0.196 & 0.238 & 0.264 & 0.213 & $1.2 \pm 4.0$ & $0.22 \pm 0.03$ \\
\hline 308.15 & 0.300 & 0.356 & 0.374 & 0.321 & $1.2 \pm 4.6$ & $0.33 \pm 0.04$ \\
\hline 318.15 & 0.440 & 0.496 & 0.485 & 0.474 & $1.8 \pm 3.1$ & $0.46 \pm 0.02$ \\
\hline 328.15 & 0.615 & 0.722 & 0.723 & 0.686 & $4.3 \pm 6.3$ & $0.66 \pm 0.05$ \\
\hline 338.15 & 0.893 & 1.007 & 0.962 & 0.968 & $3.8 \pm 6.0$ & $0.93 \pm 0.05$ \\
\hline 348.15 & 1.280 & 1.304 & 1.281 & 1.344 & $4.6 \pm 2.6$ & $1.27 \pm 0.02$ \\
\hline 358.15 & 1.371 & 1.738 & 1.610 & 1.737 & $22.9 \pm 17.7$ & $1.47 \pm 0.14$ \\
\hline \multicolumn{7}{|c|}{$x_{1}=0.424, x_{2}=0.576$} \\
\hline$w / 10^{-6}$ & 1235 & 3632 & 8847 & 12971 & & \\
\hline 298.15 & 0.238 & 0.237 & 0.267 & 0.251 & $1.5 \pm 1.6$ & $0.24 \pm 0.01$ \\
\hline 308.15 & 0.364 & 0.365 & 0.389 & 0.373 & $1.1 \pm 1.4$ & $0.37 \pm 0.01$ \\
\hline 318.15 & 0.533 & 0.521 & 0.515 & 0.546 & $1.1 \pm 1.7$ & $0.52 \pm 0.01$ \\
\hline
\end{tabular}




\begin{tabular}{|c|c|c|c|c|c|c|}
\hline 328.15 & 0.745 & 0.780 & 0.791 & 0.787 & $3.2 \pm 1.9$ & $0.76 \pm 0.01$ \\
\hline 338.15 & 1.080 & 1.064 & 1.076 & 1.093 & $1.6 \pm 1.3$ & $1.07 \pm 0.01$ \\
\hline 348.15 & 1.549 & 1.469 & 1.465 & 1.505 & $-2.5 \pm 5.1$ & $1.51 \pm 0.04$ \\
\hline 358.15 & 1.659 & 2.000 & 1.874 & 1.932 & $15.7 \pm 17.2$ & $1.77 \pm 0.13$ \\
\hline \multicolumn{7}{|c|}{$x_{1}=0.620, x_{2}=0.380$} \\
\hline$w / 10^{-6}$ & 1462 & 3638 & 8081 & 14417 & & \\
\hline 298.15 & 0.267 & 0.294 & 0.278 & 0.284 & $0.7 \pm 1.5$ & $0.28 \pm 0.01$ \\
\hline 308.15 & 0.407 & 0.426 & 0.409 & 0.419 & $0.5 \pm 1.2$ & $0.41 \pm 0.01$ \\
\hline 318.15 & 0.576 & 0.560 & 0.545 & 0.616 & $3.4 \pm 3.6$ & $0.55 \pm 0.03$ \\
\hline 328.15 & 0.853 & 0.855 & 0.848 & 0.886 & $2.7 \pm 1.5$ & $0.84 \pm 0.01$ \\
\hline 338.15 & 1.154 & 1.156 & 1.164 & 1.244 & $7.5 \pm 2.7$ & $1.13 \pm 0.02$ \\
\hline 348.15 & 1.582 & 1.565 & 1.597 & 1.719 & $12.2 \pm 4.5$ & $1.54 \pm 0.03$ \\
\hline 358.15 & 2.137 & 1.994 & 2.057 & 2.212 & $9.5 \pm 11.4$ & $2.04 \pm 0.09$ \\
\hline \multicolumn{7}{|c|}{$x_{1}=0.661, x_{2}=0.339$} \\
\hline$w / 10^{-6}$ & 1517 & 4631 & 8906 & & & \\
\hline 298.15 & 0.271 & 0.279 & 0.286 & & $2.2 \pm 0.2$ & $0.27 \pm 0.01$ \\
\hline 308.15 & 0.415 & 0.419 & 0.426 & & $1.7 \pm 0.2$ & $0.41 \pm 0.01$ \\
\hline 318.15 & 0.591 & 0.647 & 0.625 & & $4.8 \pm 7.2$ & $0.6 \pm 0.04$ \\
\hline 328.15 & 0.879 & 0.903 & 0.898 & & $2.6 \pm 2.7$ & $0.88 \pm 0.01$ \\
\hline 338.15 & 1.195 & 1.285 & 1.257 & & $8.6 \pm 10.9$ & $1.21 \pm 0.05$ \\
\hline 348.15 & 1.645 & 1.756 & 1.733 & & $12.5 \pm 12.7$ & $1.66 \pm 0.06$ \\
\hline 358.15 & 2.232 & 2.292 & 2.344 & & $17.0 \pm 1.9$ & $2.22 \pm 0.01$ \\
\hline \multicolumn{7}{|c|}{$x_{1}=0.752, x_{2}=0.248$} \\
\hline$w / 10^{-6}$ & 1651 & 5096 & 10593 & 13132 & & \\
\hline 298.15 & 0.303 & 0.309 & 0.406 & 0.330 & $3.7 \pm 6.0$ & $0.31 \pm 0.05$ \\
\hline 308.15 & 0.452 & 0.465 & 0.590 & 0.469 & $3.3 \pm 8.7$ & $0.47 \pm 0.07$ \\
\hline 318.15 & 0.632 & 0.676 & 0.779 & 0.657 & $3.1 \pm 8.7$ & $0.67 \pm 0.07$ \\
\hline 328.15 & 0.921 & 0.977 & 1.197 & 0.908 & $1.7 \pm 18.6$ & $0.99 \pm 0.14$ \\
\hline 338.15 & 1.231 & 1.358 & 1.626 & 1.214 & $1.0 \pm 26.4$ & $1.35 \pm 0.20$ \\
\hline 348.15 & 1.666 & 1.873 & 2.211 & 1.612 & $-2.1 \pm 37.7$ & $1.85 \pm 0.29$ \\
\hline 358.15 & 2.223 & 2.407 & 2.826 & 2.011 & $-13.7 \pm 47.1$ & $2.45 \pm 0.36$ \\
\hline
\end{tabular}


Table S6: Least Linear Square Fit Parameters of Density Temperature Dependence of Various Polydisperse Polyethylene Glycol (PEG) at Ambient Pressure $(0.10 \pm 0.01 \mathrm{MPa})$.

\begin{tabular}{llrr} 
PEG & $x_{1}{ }^{\mathrm{a}}$ & slope $/ \mathrm{kg} \cdot \mathrm{m}^{-3} \cdot \mathrm{K}^{-1}$ & intercept $/ \mathrm{kg} \cdot \mathrm{m}^{-3}$ \\
\hline PEG200 (ACROS) & N/A & $-0.7974 \pm 0.0004$ & $1358.6 \pm 0.1$ \\
PEG200 (Rigaku) & N/A & $-0.8047 \pm 0.0020$ & $1360.7 \pm 0.6$ \\
PEG400 (ACROS) & N/A & $-0.8177 \pm 0.0011$ & $1368.2 \pm 0.3$ \\
Binary Mixture & 0.197 & $-0.8088 \pm 0.0006$ & $1362.7 \pm 0.2$ \\
Binary Mixture & 0.424 & $-0.8054 \pm 0.0006$ & $1361.7 \pm 0.2$ \\
Binary Mixture & 0.620 & $-0.8001 \pm 0.0005$ & $1360.0 \pm 0.2$ \\
Binary Mixture & 0.661 & $-0.8002 \pm 0.0019$ & $1360.2 \pm 0.6$ \\
Binary Mixture & 0.752 & $-0.7977 \pm 0.0010$ & $1359.1 \pm 0.3$ \\
\hline
\end{tabular}

${ }^{a}$ Mole fraction of triethyleneglycol with hexaethyleneglycol being the second component. 
Table S7: Arrhenius Fit Coefficients for the Viscosity Temperature Dependence at Ambient Pressure (at $0.10 \pm 0.01 \mathrm{MPa}$ )

\begin{tabular}{lrrr}
\hline PEG & $x_{1}{ }^{\mathrm{a}}$ & $\ln (A / \mathrm{mPa} \cdot \mathrm{s})$ & $E_{a} / \mathrm{kJ}^{\mathrm{m}} \mathrm{mol}^{-1}$ \\
\hline PEG200 (ACROS) & N/A & $-9.09 \pm 0.21$ & $32.2 \pm 0.6$ \\
PEG200 (Rigaku) & N/A & $-9.12 \pm 0.26$ & $32.3 \pm 0.7$ \\
PEG400 (ACROS) & N/A & $-8.37 \pm 0.30$ & $31.8 \pm 0.8$ \\
Binary Mixture & 0.197 & $-8.99 \pm 0.19$ & $32.4 \pm 0.5$ \\
Binary Mixture & 0.424 & $-9.04 \pm 0.24$ & $32.3 \pm 0.7$ \\
Binary Mixture & 0.620 & $-9.05 \pm 0.17$ & $32.1 \pm 0.5$ \\
Binary Mixture & 0.661 & $-9.12 \pm 0.29$ & $32.2 \pm 0.8$ \\
Binary Mixture & 0.752 & $-9.14 \pm 0.24$ & $32.1 \pm 0.6$ \\
\hline
\end{tabular}

${ }^{a}$ Mole fraction of triethylene glycol with hexaethylene glycol being the second component 
Table S8: Arrhenius Fit Coefficients for the Self-Diffusion Coefficient Temperature Dependence at Ambient Pressure (at $0.10 \pm 0.01 \mathrm{MPa}$ )

\begin{tabular}{lrrr}
\hline PEG & $x_{1}{ }^{\mathrm{a}}$ & $\ln \left(\mathrm{A} / \mathrm{m}^{2} \cdot \mathrm{s}^{-1}\right)$ & $E_{a} / \mathrm{kJ} \cdot \mathrm{mol}^{-1}$ \\
\hline PEG200 (ACROS) & N/A & $10.27 \pm 0.07$ & $-28.5 \pm 0.2$ \\
PEG200 (Rigaku) & N/A & $10.69 \pm 0.39$ & $-29.6 \pm 1.1$ \\
PEG400 (ACROS) & N/A & $8.61 \pm 0.08$ & $-26.2 \pm 0.2$ \\
Binary Mixture & 0.197 & $10.18 \pm 0.30$ & $-28.9 \pm 0.8$ \\
Binary Mixture & 0.424 & $10.87 \pm 0.29$ & $-30.4 \pm 0.8$ \\
Binary Mixture & 0.620 & $10.68 \pm 0.17$ & $-29.7 \pm 0.5$ \\
Binary Mixture & 0.661 & $11.28 \pm 0.08$ & $-31.2 \pm 0.2$ \\
Binary Mixture & 0.752 & $11.17 \pm 0.10$ & $-30.5 \pm 0.3$ \\
\hline
\end{tabular}

${ }^{\text {a }}$ Mole fraction of triethylene glycol with hexaethylene glycol being the second component 
Table S9: Exact Masses, $m$, and Mass Fractions, $w$, of Binary Tri- (1) and Hexaethylene Glycol (2) Samples

\begin{tabular}{|c|c|c|c|c|c|}
\hline$x_{1}^{\mathrm{a}}$ & 0.197 & 0.424 & 0.620 & 0.661 & 0.752 \\
\hline $\mathrm{AMW}^{\mathrm{b}}$ & 256.30 & 226.30 & 200.39 & 194.98 & 182.95 \\
\hline & \multicolumn{5}{|c|}{ no water addition } \\
\hline$m_{1} / \mathrm{g}$ & $1.511 \pm 0.001$ & $3.434 \pm 0.001$ & $5.732 \pm 0.001$ & $6.042 \pm 0.001$ & $3.756 \pm 0.001$ \\
\hline$m_{2} / \mathrm{g}$ & $11.593 \pm 0.001$ & $8.782 \pm 0.001$ & $6.606 \pm 0.001$ & $9.737 \pm 0.001$ & $3.625 \pm 0.001$ \\
\hline$m_{\text {water }} / \mathrm{g}$ & $0.013 \pm 0.001$ & $0.015 \pm 0.002$ & $0.018 \pm 0.002$ & $0.024 \pm 0.002$ & $0.012 \pm 0.001$ \\
\hline$w_{\text {lactual }}$ & $0.1152 \pm 0.0001$ & $0.2808 \pm 0.0001$ & $0.4639 \pm 0.0001$ & $0.3823 \pm 0.0001$ & $0.5081 \pm 0.0001$ \\
\hline$w_{\text {2actual }}$ & $0.8838 \pm 0.0001$ & $0.718 \pm 0.0001$ & $0.5346 \pm 0.0001$ & $0.6162 \pm 0.0001$ & $0.4903 \pm 0.0001$ \\
\hline$w_{\text {actual }}$ & $0.001 \pm 0.0001$ & $0.0012 \pm 0.0001$ & $0.0015 \pm 0.0001$ & $0.0015 \pm 0.0002$ & $0.0016 \pm 0.0002$ \\
\hline$x_{1 \text { actual }}$ & $0.194 \pm 0.001$ & $0.417 \pm 0.001$ & $0.61 \pm 0.001$ & $0.529 \pm 0.001$ & $0.649 \pm 0.001$ \\
\hline$x_{\text {2actual }}$ & $0.792 \pm 0.001$ & $0.568 \pm 0.001$ & $0.374 \pm 0.001$ & $0.453 \pm 0.001$ & $0.333 \pm 0.001$ \\
\hline \multirow[t]{2}{*}{$x_{\text {wactual }}$} & $0.014 \pm 0.001$ & $0.015 \pm 0.002$ & $0.016 \pm 0.002$ & $0.017 \pm 0.002$ & $0.018 \pm 0.002$ \\
\hline & \multicolumn{5}{|c|}{ after $1^{\text {st }}$ water addition } \\
\hline$m_{1} / \mathrm{g}$ & $1.264 \pm 0.001$ & $2.775 \pm 0.001$ & $4.607 \pm 0.001$ & $1.964 \pm 0.001$ & $4.438 \pm 0.001$ \\
\hline$m_{2} / g$ & $9.695 \pm 0.001$ & $7.095 \pm 0.001$ & $5.31 \pm 0.001$ & $3.166 \pm 0.001$ & $4.283 \pm 0.001$ \\
\hline$m_{\text {water }} / \mathrm{g}$ & $0.043 \pm 0.004$ & $0.036 \pm 0.004$ & $0.036 \pm 0.004$ & $0.024 \pm 0.002$ & $0.045 \pm 0.004$ \\
\hline$w_{\text {lactual }}$ & $0.1149 \pm 0.0001$ & $0.2801 \pm 0.0001$ & $0.4629 \pm 0.0002$ & $0.3811 \pm 0.0002$ & $0.5063 \pm 0.0003$ \\
\hline$w_{\text {2actual }}$ & $0.8812 \pm 0.0004$ & $0.7163 \pm 0.0003$ & $0.5335 \pm 0.0002$ & $0.6142 \pm 0.0003$ & $0.4886 \pm 0.0003$ \\
\hline$w_{\text {actual }}$ & $0.0039 \pm 0.0004$ & $0.0036 \pm 0.0004$ & $0.0036 \pm 0.0004$ & $0.0046 \pm 0.0005$ & $0.0051 \pm 0.0005$ \\
\hline$x_{1 \text { actual }}$ & $0.186 \pm 0.001$ & $0.405 \pm 0.002$ & $0.596 \pm 0.002$ & $0.511 \pm 0.003$ & $0.626 \pm 0.003$ \\
\hline$x_{\text {2actual }}$ & $0.761 \pm 0.004$ & $0.551 \pm 0.002$ & $0.365 \pm 0.001$ & $0.438 \pm 0.002$ & $0.321 \pm 0.002$ \\
\hline \multirow[t]{2}{*}{$x_{\text {wactual }}$} & $0.053 \pm 0.005$ & $0.044 \pm 0.004$ & $0.039 \pm 0.004$ & $0.052 \pm 0.005$ & $0.053 \pm 0.005$ \\
\hline & \multicolumn{5}{|c|}{ after $2^{\text {nd }}$ water addition } \\
\hline$m_{1} / \mathrm{g}$ & $1.002 \pm 0.001$ & $2.163 \pm 0.001$ & $3.304 \pm 0.001$ & $2.174 \pm 0.001$ & $3.579 \pm 0.001$ \\
\hline$m_{2} / g$ & $7.688 \pm 0.001$ & $5.531 \pm 0.001$ & $3.807 \pm 0.001$ & $3.504 \pm 0.001$ & $3.454 \pm 0.001$ \\
\hline$m_{\text {water }} / \mathrm{g}$ & $0.067 \pm 0.007$ & $0.069 \pm 0.007$ & $0.058 \pm 0.006$ & $0.051 \pm 0.005$ & $0.075 \pm 0.008$ \\
\hline$w_{\text {lactual }}$ & $0.1144 \pm 0.0001$ & $0.2786 \pm 0.0003$ & $0.4608 \pm 0.0004$ & $0.3795 \pm 0.0004$ & $0.5035 \pm 0.0005$ \\
\hline$w_{\text {2actual }}$ & $0.878 \pm 0.0007$ & $0.7125 \pm 0.0006$ & $0.5311 \pm 0.0004$ & $0.6116 \pm 0.0006$ & $0.4859 \pm 0.0005$ \\
\hline$w_{\text {actual }}$ & $0.0076 \pm 0.0008$ & $0.0089 \pm 0.0009$ & $0.0081 \pm 0.0008$ & $0.0089 \pm 0.0009$ & $0.0106 \pm 0.001$ \\
\hline$x_{1 \text { actual }}$ & $0.177 \pm 0.002$ & $0.381 \pm 0.004$ & $0.568 \pm 0.005$ & $0.487 \pm 0.005$ & $0.592 \pm 0.006$ \\
\hline$x_{\text {2actual }}$ & $0.724 \pm 0.007$ & $0.518 \pm 0.005$ & $0.348 \pm 0.003$ & $0.418 \pm 0.004$ & $0.304 \pm 0.003$ \\
\hline \multirow[t]{2}{*}{$x_{\text {wactual }}$} & $0.098 \pm 0.009$ & $0.101 \pm 0.009$ & $0.083 \pm 0.008$ & $0.095 \pm 0.009$ & $0.104 \pm 0.009$ \\
\hline & \multicolumn{5}{|c|}{ after $3^{\text {rd }}$ water addition } \\
\hline$m_{1} / g$ & $0.713 \pm 0.001$ & $1.738 \pm 0.001$ & $2.528 \pm 0.001$ & & $2.309 \pm 0.001$ \\
\hline$m_{2} / g$ & $5.47 \pm 0.001$ & $4.445 \pm 0.001$ & $2.913 \pm 0.001$ & & $2.228 \pm 0.001$ \\
\hline$m_{\text {water }} / \mathrm{g}$ & $0.08 \pm 0.008$ & $0.08 \pm 0.008$ & $0.08 \pm 0.008$ & & $0.060 \pm 0.006$ \\
\hline$w_{\text {lactual }}$ & $0.1138 \pm 0.0002$ & $0.2775 \pm 0.0004$ & $0.4579 \pm 0.0007$ & & $0.5022 \pm 0.0007$ \\
\hline$w_{\text {2actual }}$ & $0.8733 \pm 0.0011$ & $0.7097 \pm 0.0009$ & $0.5277 \pm 0.0008$ & & $0.4847 \pm 0.0007$ \\
\hline
\end{tabular}




\begin{tabular}{lrrrr}
$w_{\text {actual }}$ & $0.0128 \pm 0.0013$ & $0.0128 \pm 0.0013$ & $0.0144 \pm 0.0014$ & $0.0131 \pm 0.0013$ \\
$x_{\text {1actual }}$ & $0.166 \pm 0.003$ & $0.364 \pm 0.005$ & $0.533 \pm 0.007$ & $0.578 \pm 0.007$ \\
$x_{\text {2actual }}$ & $0.678 \pm 0.011$ & $0.496 \pm 0.007$ & $0.327 \pm 0.005$ & $0.297 \pm 0.004$ \\
$x_{\text {wactual }}$ & $0.156 \pm 0.013$ & $0.140 \pm 0.012$ & $0.140 \pm 0.012$ & $0.126 \pm 0.011$ \\
\hline
\end{tabular}

${ }^{a}$ Mole fractions of triethylene glycol based on weights of tri- and hexaethylene glycol alone with standard uncertainty of 0.001 . As a side note, the uncertainties of $x_{\text {1actual }}, x_{2 \text { actual }}$, and $x_{\text {wactual }}$ increase with water content because the relative standard uncertainty of the mass of water is $10 \%$. However, the increased mole fraction uncertainty is essentially irrelevant for the density, viscosity and self-diffusion coefficient measurement uncertainties because of the observed near independence of these quantities on the sample water content for the PEG systems.

${ }^{\mathrm{b}}$ Average molar weight based on tri- and hexaethylene glycol components only. 
Table S10: Molar Volumes in $\mathrm{mL}$ at $0.10 \pm 0.01 \mathrm{MPa}$ of Ethylene Glycol Oligomers, $\mathrm{H}\left(\mathrm{OC}_{2} \mathrm{H}_{4}\right)_{\mathrm{n}} \mathrm{OH}$, From Densities Reported by Hoffmann et al. ${ }^{1}$ and Linear Least Squares Fitting of Their Temperature Dependences ${ }^{\mathrm{a}}$

\begin{tabular}{lrrrrrrrr}
\hline $\mathrm{T} / \mathrm{K}$ & $\mathrm{n}=$ & 3 & 4 & 5 & 6 & 7 & 8 & 9 \\
\hline 298.15 & 95.28 & 134.00 & 173.41 & 212.43 & 251.11 & 291.09 & 330.29 & 368.87 \\
308.15 & 95.91 & 134.95 & 174.66 & 213.98 & 253.02 & 293.18 & 332.70 & 371.59 \\
318.15 & 96.55 & 135.90 & 175.93 & 215.54 & 254.94 & 295.30 & 335.14 & 374.32 \\
328.15 & 97.21 & 136.88 & 177.21 & 217.13 & 256.82 & 297.44 & 337.63 & 377.11 \\
338.15 & 97.88 & 137.88 & 178.52 & 218.73 & 258.77 & 299.66 & 340.14 & 379.94 \\
348.15 & 98.56 & 138.89 & 179.85 & 220.38 & 260.71 & 301.89 & 342.68 & 382.76 \\
358.15 & 99.26 & 139.93 & 181.19 & 222.05 & 262.70 & 304.19 & 345.28 & 385.59 \\
Slope/ & 0.0663 & 0.0988 & 0.1297 & 0.1602 & 0.1928 & 0.2182 & 0.2497 & 0.2791 \\
$\mathrm{~mL} \cdot \mathrm{mol}^{-1} \cdot \mathrm{K}^{-1}$ & \pm 0.0005 & \pm 0.0007 & \pm 0.0007 & \pm 0.0010 & \pm 0.0006 & \pm 0.0016 & \pm 0.0014 & \pm 0.0010 \\
Intercept/ $_{\mathrm{mL} \cdot \mathrm{mol}^{-1}}$ & 75.48 & 104.51 & 134.68 & 164.60 & 193.61 & 225.94 & 255.76 & 285.59 \\
\hline
\end{tabular}

a Standard uncertainty of molar volumes estimated to be $0.20 \mathrm{~mL}$ based on stated relative density standard uncertainty of $0.1 \%$. 

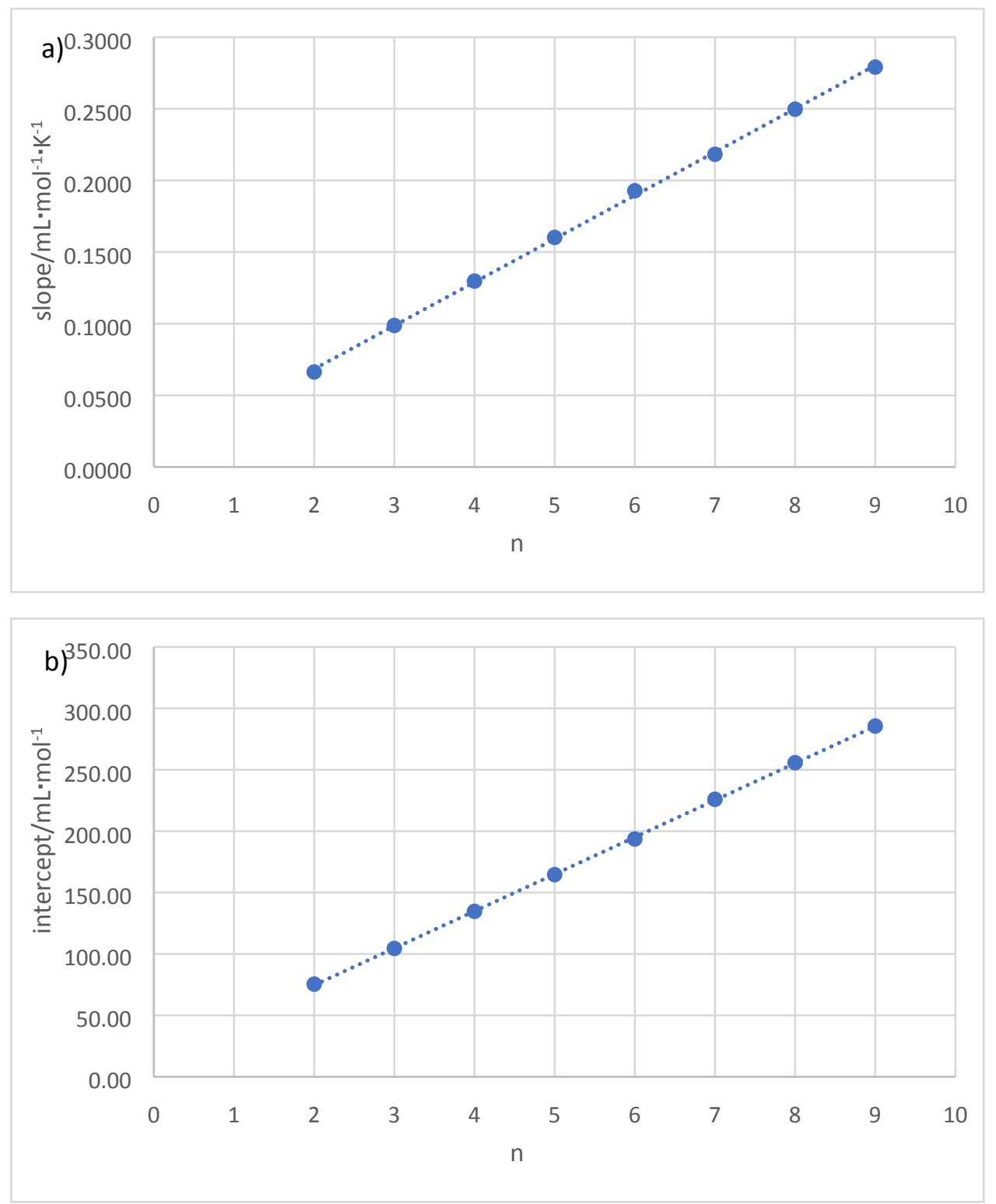

Figure S2. Slope (a) and intercepts (b) of the linear dependence of temperature dependence with respect to ethylene oxide repeat unit, $\mathrm{n}$, of molar volume of ethylene glycol oligomers, $\mathrm{H}\left(\mathrm{OC}_{2} \mathrm{H}_{4}\right)_{\mathrm{n}} \mathrm{OH}$. Linear dependences are evident with slope $(n)=(0.0303 \pm 0.0003) n+(0.0079 \pm 0.0017)$ and intercept $(n)=$ $(30.11 \pm 0.12) \mathrm{n}+(14.38 \pm 0.74)$. 
Table S11: Molar Volumes at 0.10 $\pm 0.01 \mathrm{MPa}$ of Tri-(1) and Hexaethylene (2) Glycol Binary Mixtures and PEG200 and Linear Least Squares Fitting of Their Temperature Dependence ${ }^{\text {a }}$

\begin{tabular}{|c|c|c|c|c|c|c|c|c|c|}
\hline$T / \mathrm{K}$ & 0.000 & 0.197 & 0.424 & 0.620 & 0.661 & 0.752 & 1.000 & $\begin{array}{l}\text { PEG200 } \\
\text { Acros }\end{array}$ & $\begin{array}{l}\text { PEG200 } \\
\text { Rigaku }\end{array}$ \\
\hline 298.15 & 251.11 & 228.49 & 201.75 & 178.70 & 173.83 & 163.16 & 134.00 & 183.65 & 188.58 \\
\hline 308.15 & 253.02 & 230.16 & 203.21 & 179.97 & 175.07 & 164.34 & 134.95 & 184.97 & 189.96 \\
\hline 318.15 & 254.94 & 231.84 & 204.69 & 181.28 & 176.33 & 165.51 & 135.90 & 186.30 & 191.32 \\
\hline 328.15 & 256.82 & 233.56 & 206.20 & 182.60 & 177.63 & 166.70 & 136.88 & 187.66 & 192.72 \\
\hline 338.15 & 258.77 & 235.29 & 207.72 & 183.93 & 178.94 & 167.93 & 137.88 & 189.03 & 194.13 \\
\hline 348.15 & 260.71 & 237.04 & 209.27 & 185.30 & 180.23 & 169.18 & 138.89 & 190.43 & 195.63 \\
\hline 358.15 & 262.70 & 238.83 & 210. & 186.69 & 181.62 & 170.44 & 139.93 & 191.85 & 197.07 \\
\hline Slope/ & 0.1928 & 0.1722 & 0.1514 & 0.1332 & 0.1296 & 0.1212 & 0.0988 & 0.1365 & 0.1415 \\
\hline $\mathrm{mL} \cdot \mathrm{mol}^{-1} \cdot \mathrm{K}^{-1}$ & \pm 0.0006 & \pm 0.0008 & \pm 0.0008 & \pm 0.0008 & \pm 0.0009 & \pm 0.0008 & \pm 0.0007 & \pm 0.0008 & \pm 0.0010 \\
\hline Intercept/ & 193.61 & 177.10 & 156.57 & 138.93 & 135.15 & 126.96 & 104.51 & 142.92 & 146.34 \\
\hline $\mathrm{mL} \cdot \mathrm{mol}^{-1}$ & \pm 0.21 & \pm 0.29 & \pm 0.26 & \pm 0.27 & \pm 0.30 & \pm 0.25 & \pm 0.23 & \pm 0.29 & \pm 0.33 \\
\hline
\end{tabular}

a Standard uncertainty of molar volumes estimated to be $0.20 \mathrm{~mL}$ based on relative density standard uncertainty of $0.1 \%$. Standard uncertainty of temperature is $0.02 \mathrm{~K}$. 
Table S12: Excess Molar Volumes MPa of Tri- (1) and Hexaethylene Glycol (2) Binary Mixtures and PEG200 as calculated from entries in Tables S9 and S10. ${ }^{\text {a }}$

\begin{tabular}{rrrrrrrrrr}
\hline$x_{1}$ & 0.000 & 0.197 & 0.424 & 0.620 & 0.661 & 0.752 & 1.000 & $\begin{array}{r}\text { PEG200 } \\
\text { Acros }\end{array}$ & $\begin{array}{r}\text { PEG200 } \\
\text { Rigaku }\end{array}$ \\
298.15 & & & & & & & & 0.101 \\
308.15 & 0.000 & 0.454 & 0.297 & 0.194 & 0.135 & 0.112 & 0.000 & 0.060 & 0.101 \\
318.15 & 0.000 & 0.404 & 0.257 & 0.159 & 0.094 & 0.108 & 0.000 & 0.048 & 0.108 \\
328.15 & 0.000 & 0.367 & 0.239 & 0.138 & 0.089 & 0.078 & 0.000 & 0.026 & 0.089 \\
338.15 & 0.000 & 0.337 & 0.205 & 0.117 & 0.078 & 0.075 & 0.000 & 0.011 & 0.072 \\
348.15 & 0.000 & 0.330 & 0.216 & 0.121 & 0.041 & 0.075 & 0.000 & 0.002 & 0.123 \\
358.15 & 0.000 & 0.317 & 0.188 & 0.107 & 0.068 & 0.060 & 0.000 & -0.012 & 0.091 \\
\hline
\end{tabular}

${ }^{a}$ Standard uncertainty of excess molar volumes estimated to be $0.50 \mathrm{~mL}$ based. Standard uncertainty of temperature is $0.02 \mathrm{~K}$. 
Table S13: Excess Molar Volumes in $\mathrm{mL} \cdot \mathrm{mol}^{-1}$ of Tri- (1) and Hexaethylene Glycol (2) Binary Mixtures as calculated from fits in eqs. 3 and $4{ }^{\text {a }}$

\begin{tabular}{rrrrrrrr}
\hline$x_{1}$ & 0.000 & 0.197 & 0.424 & 0.620 & 0.661 & 0.752 & 1.000 \\
$T / \mathrm{K}$ & & & & & & & \\
298.15 & -0.249 & -0.222 & -0.191 & -0.164 & -0.158 & -0.146 & -0.112 \\
308.15 & -0.228 & -0.206 & -0.182 & -0.160 & -0.156 & -0.146 & -0.118 \\
318.15 & -0.207 & -0.191 & -0.172 & -0.156 & -0.153 & -0.145 & -0.125 \\
328.15 & -0.187 & -0.176 & -0.163 & -0.153 & -0.150 & -0.145 & -0.132 \\
338.15 & -0.166 & -0.161 & -0.154 & -0.149 & -0.148 & -0.145 & -0.138 \\
348.15 & -0.145 & -0.145 & -0.145 & -0.145 & -0.145 & -0.145 & -0.145 \\
358.15 & -0.125 & -0.130 & -0.136 & -0.141 & -0.142 & -0.145 & -0.151 \\
\hline
\end{tabular}

a Standard uncertainty of excess molar volumes estimated to be $0.50 \mathrm{~mL}$ based. Standard uncertainty of temperature is $0.02 \mathrm{~K}$. 
Table S14: Calculated Viscosities in mPa's of Tri- (1) and Hexaethylene Glycol (2) Binary Mixtures Based on $E_{\mathrm{a}}$ and $\ln A$ Parameters of Ethylene Glycol Oligomers from Hoffmann et al. ${ }^{1}$

\begin{tabular}{rrrrrrrr}
\hline$x_{1}$ & 0.197 & 0.424 & 0.62 & 0.661 & 0.752 & $\begin{array}{r}\text { PEG200 } \\
\text { Acros }\end{array}$ & $\begin{array}{r}\text { PEG200 } \\
\text { Rigaku }\end{array}$ \\
298.15 & & & & & & 44.75 & 45.85 \\
308.15 & 55.47 & 48.94 & 43.92 & 42.94 & 40.83 & 44.75 \\
318.15 & 36.81 & 32.61 & 29.37 & 28.73 & 27.37 & 29.91 & 30.62 \\
328.15 & 25.07 & 22.29 & 20.14 & 19.72 & 18.81 & 20.50 & 20.97 \\
338.15 & 17.48 & 15.60 & 14.14 & 13.85 & 13.23 & 14.38 & 14.70 \\
348.15 & 12.44 & 11.14 & 10.13 & 9.93 & 9.50 & 10.30 & 10.52 \\
358.15 & 9.04 & 8.12 & 7.40 & 7.26 & 6.95 & 7.52 & 7.68 \\
\hline
\end{tabular}


Table S15: Calculated Self-Diffusion Coefficients in $10^{-10} \mathrm{~m}^{2} \cdot \mathrm{s}^{-1}$ of Tri- (1) and Hexaethylene Glycol (2) Binary Mixtures Based on $E_{\mathrm{a}}$ and $\ln A$ Parameters of Ethylene Glycol Oligomers from Hoffmann et al. ${ }^{1}$

\begin{tabular}{rccccccc}
\hline$x_{1}$ & 0.197 & 0.424 & 0.62 & 0.661 & 0.752 & $\begin{array}{c}\text { PEG200 } \\
\text { Acros }\end{array}$ & $\begin{array}{c}\text { PEG200 } \\
\text { Rigaku }\end{array}$ \\
298.15 & & & & & & 0.316 & 0.309 \\
308.15 & 0.255 & 0.289 & 0.322 & 0.330 & 0.347 & 0.316 \\
318.15 & 0.385 & 0.434 & 0.482 & 0.493 & 0.517 & 0.473 & 0.462 \\
328.15 & 0.565 & 0.635 & 0.703 & 0.718 & 0.752 & 0.690 & 0.675 \\
338.15 & 0.810 & 0.908 & 1.001 & 1.022 & 1.070 & 0.984 & 0.963 \\
348.15 & 1.137 & 1.270 & 1.397 & 1.426 & 1.490 & 1.374 & 1.345 \\
358.15 & 1.566 & 1.744 & 1.913 & 1.950 & 2.036 & 1.882 & 1.844 \\
\hline
\end{tabular}


Table S16: Differences of Calculated (Table S13) and Measured Viscosities in $\mathrm{mPa} \cdot \mathrm{s}$ (Intercepts Listed in Table 7)

\begin{tabular}{rrrrrrrr}
\hline$x_{1}{ }^{\mathrm{a}}$ & 0.197 & 0.424 & 0.62 & 0.661 & 0.752 & $\begin{array}{c}\text { PEG200 } \\
\text { Acros }\end{array}$ & $\begin{array}{r}\text { PEG200 } \\
\text { Rigaku }\end{array}$ \\
298.15 & -6.81 & -7.41 & -7.01 & -7.03 & -6.41 & -7.58 & -6.10 \\
308.15 & -1.93 & -2.02 & -2.55 & -2.14 & -1.87 & -2.50 & -0.95 \\
318.15 & -0.51 & -0.51 & -1.13 & -0.61 & -0.65 & -0.99 & 0.23 \\
328.15 & -0.11 & -0.13 & -0.52 & -0.17 & -0.21 & -0.34 & 0.31 \\
338.15 & -0.24 & -0.21 & -0.41 & -0.28 & -0.25 & -0.31 & 0.16 \\
348.15 & -0.45 & -0.52 & -0.50 & -0.69 & -0.42 & -0.46 & -0.15 \\
358.15 & -0.04 & -0.13 & -0.15 & 0.06 & -0.08 & -0.18 & 0.05 \\
\hline a & & & & & & \\
\hline
\end{tabular}

${ }^{a}$ Mole fraction of triethylene glycol with hexaethylene glycol being the second component 
Table S17: Differences of Calculated (Table S14) and Measured Self-Diffusion Coefficients in $10^{-10} \mathrm{~m}^{2} \cdot \mathrm{s}^{-1}$ (Intercepts Listed in Table S5).

\begin{tabular}{rrrrrrrr}
\hline$x_{1}{ }^{\mathrm{a}}$ & 0.197 & 0.424 & 0.62 & 0.661 & 0.752 & $\begin{array}{r}\text { PEG200 } \\
\text { Acros }\end{array}$ & $\begin{array}{r}\text { PEG200 } \\
\text { Rigaku }\end{array}$ \\
298.15 & & & & & & 0.03 & 0.03 \\
308.15 & 0.03 & 0.05 & 0.05 & 0.06 & 0.03 & 0.03 & 0.06 \\
318.15 & 0.05 & 0.07 & 0.07 & 0.08 & 0.04 & 0.06 & 0.04 \\
328.15 & 0.10 & 0.11 & 0.15 & 0.12 & 0.09 & 0.09 & 0.07 \\
338.15 & 0.15 & 0.15 & 0.16 & 0.14 & 0.08 & 0.16 & 0.12 \\
348.15 & 0.20 & 0.20 & 0.27 & 0.22 & 0.14 & 0.24 & 0.13 \\
358.15 & 0.29 & 0.23 & 0.37 & 0.29 & 0.18 & 0.38 & 0.13 \\
\hline
\end{tabular}

${ }^{a}$ Mole fraction of triethylene glycol with hexaethylene glycol being the second component 
Bibliography of references cited in supplementary materials.

1. Hoffmann, M. M.; Horowitz, R. H.; Gutmann, T.; Buntkowsky, G., Densities, Viscosities, and SelfDiffusion Coefficients of Ethylene Glycol Oligomers. J. Chem. Eng. Data 2021, 66, 2480-2500.

2. Carvalho, P. J.; Fonseca, C. H. G.; Moita, M.-L. C. J.; Santos, Â. F. S.; Coutinho, J. A. P., Thermophysical Properties of Glycols and Glymes. J. Chem. Eng. Data 2015, 60, 3721-3737.

3. Hao, C.; Zhao, L.; Yue, X.; Pang, Y.; Zhang, J., Density, Dynamic Viscosity, Excess Properties and Intermolecular Interaction of Triethylene Glycol + N,N-Dimethylformamide Binary Mixture. J. Mol. Liq. 2019, 274, 730-739.

4. Qiao, X.; Zhao, T.; Guo, B.; Sha, F.; Zhang, F.; Xie, X.; Zhang, J.; Wei, X., Excess Properties and Spectral Studies for Binary System Tri-Ethylene Glycol + Dimethyl Sulfoxide. J. Mol. Liq. 2015, 212, 187-195.

5. Begum, S. K.; Clarke, R. J.; Ahmed, M. S.; Begum, S.; Saleh, M. A., Densities, Viscosities, and Surface Tensions of the System Water + Diethylene Glycol. J. Chem. Eng. Data 2011, 56, 303-306.

6. Guo, Z.; Zhang, J.; Zhang, T.; Li, C.; Zhang, Y.; Bai, J., Liquid Viscosities, Excess Properties, and Viscous Flow Thermodynamics of Triethylene Glycol + Water Mixtures at $\mathrm{T}=(298.15,303.15$, 308.15, 313.15, and 318.15) K. J. Mol. Liq. 2012, 165, 27-31.

7. Ghaedi, H.; Ayoub, M.; Sufian, S.; Shariff, A. M.; Lal, B., The Study on Temperature Dependence of Viscosity and Surface Tension of Several Phosphonium-Based Deep Eutectic Solvents. J. Mol. Liq. 2017, 241, 500-510.

8. Almasi, M., Thermophysical and Transport Properties of Binary Mixtures Containing Triethylene Glycol and Alcohols at Different Temperatures. J. Therm. Anal. Calorim. 2016, 124, 399-405.

9. Mesquita, F. M. R.; Feitosa, F. X.; Aznar, M.; de Sant'Ana, H. B.; Santiago-Aguiar, R. S., Density, Viscosities, and Excess Properties for Binary Mixtures of Sulfolane + Alcohols and Sulfolane + Glycols at Different Temperatures. J. Chem. Eng. Data 2014, 59, 2196-2206.

10. Chen, X.; Li, H.; Guo, F., Measurement and Correlation of Densities and Viscosities of Thiourea in Triglycol Solution Using Vtf Equation. Russ. J. Phys. Chem. A 2010, 84, 2262-2267.

11. Pereira, M. F. V.; Avelino, H. M. N. T.; Caetano, F. J. P.; Fareleira, J. M. N. A., Viscosity of Liquid Diethylene, Triethylene and Tetraethylene Glycols at Moderately High Pressures Using a Vibrating Wire Instrument. Fluid Phase Equilib. 2019, 480, 87-97. 